\title{
NONLINEAR INTEGRAL EQUATIONS OF THE HAMMERSTEIN TYPE $\left({ }^{1}\right)$
}

\author{
BY \\ C. L. DOLPH
}

Introduction. Several authors have treated nonlinear integral equations of the form:

$$
\psi(x)=\int_{a}^{b} K(x, y) f[y, \psi(y)] d y
$$

where $K(x, y)$ is a continuous kernel in $L_{2}$ and $f(x, y)$ is a nonlinear function of $y$, continuous in $x$ over the interval $a \leqq x \leqq b$ and continuous in $y$ over the interval $-\infty \leqq y \leqq \infty$.

Since even the linear integral equation

$$
\psi(x)=\lambda \int_{a}^{b} K(x, y) \psi(y) d y+h(x)
$$

fails to possess a solution in general if $\lambda$ is equal to any of the characteristic values $\lambda_{i}, i=1,2,3, \cdots$, of the kernel $K(x, y)$, it is not surprising that all treatments of (1) have been limited to the cases in which equation (2) is in some sense (to be made more precise later) a majorant for (1) when $\lambda=\lambda_{1}$, the smallest characteristic value of $K(x, y)$. Thus, if $K(x, y)$ is assumed to be positive-definite and symmetric, Hammerstein $[1$, Theorem 1$]\left({ }^{2}\right)$ proved that at least one solution would always exist when $f(x, y)$ satisfied, for all $y$,

$$
2 \int_{0}^{y} f(x, s) d s \leqq \mu_{1} y^{2}+C_{1}, \quad C_{1}>0,0<\mu_{1}<\lambda_{1} .
$$

Iglisch [2, Theorem 1], using reasoning similar to that of the fixed-point method, assumed the stronger condition that there existed a $y_{0}>0$ such that for $|y|>y_{0}, f(x, y)$ satisfied the inequality:

$$
0<\frac{|f(x, y)-f(x, 0)|}{|y|} \leqq \mu_{1}<\lambda_{1}
$$

Presented to the Society, November 2, 1946; received by the editors September 8, 1945, and in revised form, April 13, 1948.

(1) Aside from minor modifications, this paper is essentially the same as the author's doctoral dissertation which was written at Princeton University under the direction of Professor H. F. Bohnenblust. The author is greatly indebted to Professor Bohnenblust for many helpful suggestions and criticism. He would also like to take this opportunity to thank his many other friends at Princeton for their interest and help.

(2) Numbers in brackets refer to the bibliography at the end of the paper. 
to establish the same result.

Hammerstein [1, Theorem 6] and Iglisch [2, Theorem 2] proved that the still stronger condition:

$$
\frac{\left|f\left(x, y_{2}\right)-f\left(x, y_{1}\right)\right|}{\left|y_{2}-y_{1}\right|} \leqq \mu_{1}<\lambda_{1}
$$

was sufficient to guarantee the existence of a unique solution. Golomb [6] generalized this last result to a system of $n$ equations of the type (1) in which the kernels $K_{i}(x, y), i=1, \cdots, n$, were unsymmetric and possessed absolutely and uniformly convergent bilinear expansions in their characteristic functions.

The conditions (A), (B), (C) are all equivalent for equation (2) and the above results are classical. Moreover, as is well known, in the linear theory it is sufficient to require $\lambda_{N}<\lambda<\lambda_{N+1}$ to guarantee the above results. In this paper similar results are derived for equation (1) when the kernel is symmetric and has a finite norm in Hilbert space.

Specifically, in part I, (B) and (C) are replaced by double inequalities involving $\lambda_{N}<\mu_{N}$ and $\mu_{N+1}<\lambda_{N+1}$ in the obvious way and used to establish the existence of an a priori bound for the norm of all possible solutions of (1) even in the non-positive-definite case. The Schauder-Leray [5] fixed-point method is then used to establish existence under (B) and hence under (C). This new a priori estimate is also sufficient to establish uniqueness under (C) by purely classical reasoning, and to generalize the Picard approximation process first used by Golomb to give a constructive proof of existence.

Part II is concerned with the corresponding generalization of (A). A simple three-dimensional example is given which illustrates the fact that there are no topological reasons necessitating the existence of an a priori estimate. Thus, while Hammerstein employed direct methods of the calculus of variations (that is, the Rayleigh-Ritz process) under (A), methods of the calculus of variations in the large are used here to establish the existence of a solution for a much more restricted class of functions than those allowed by the direct generalization of (A). Nevertheless, these methods are not without interest since they do not employ an a priori estimate at any point, but yield an estimate of the norm once the existence of a solution has been established. They therefore might well be called methods of a posteriori estimation.

Part III consists of two applications of the preceding theory to differential equations.

\section{PART I}

The main aim of this section is to investigate the existence and uniqueness of solutions to the nonlinear integral equation:

$$
\psi(x)=\int_{a}^{b} K(x, y) f[y, \psi(y)] d y
$$


under more general conditions than those previously treated. Specifically, the following theorems will be proved:

THEOREM 1.1. (a) Let $L_{2}$ be a Hilbert space whose elements are real measurable functions $h(x)$ defined over $a \leqq x \leqq b$ for which the norm

$$
\|h\|=\left\{\int_{a}^{b}|h(x)|^{2} d x\right\}^{1 / 2}
$$

is finite.

(b) Let

$$
K=\int_{a}^{b} K(x, y)[] d y
$$

be a completely continuous Hermitian operator in $L_{2}$ with (real) characteristic values, $\lambda_{j}, j=0,1, \cdots$,

$$
\cdots \leqq \lambda_{-2} \leqq \lambda_{-1}<0<\lambda_{0} \leqq \lambda_{1} \leqq \lambda_{2} \cdots .
$$

(c) Let $f(x, y)$ be a continuous function of $x$ and $y$ over the intervals $a \leqq x \leqq b$, $-\infty \leqq y \leqq \infty$ respectively, for which there exists positive constants $A, y_{0}$ and numbers $\mu_{N}, \mu_{N+1}$ in the relation $\lambda_{N}<\mu_{N}<\mu_{N+1}<\lambda_{N+1}$ so that the inequalities

$$
\begin{array}{rlrl}
\mu_{N} y-A & \leqq f(x, y) \leqq \mu_{N+1} y+A & & \text { for } y>y_{0}, \\
\mu_{N+1} y-A \leqq f(x, y) \leqq \mu_{N} y+A & & \text { for } y<y_{0}
\end{array}
$$

hold; then there exists at least one solution to equation (1.1).

THEOREM 1.2. If $f(x, y)$ satisfies the stronger inequalities

$$
\lambda_{N}<\mu_{N} \leqq \frac{f\left(x, y_{2}\right)-f\left(x, y_{1}\right)}{y_{2}-y_{1}} \leqq \mu_{N+1}<\lambda_{N+1}
$$

for all $y_{1}, y_{2}$, instead of $\left(\mathrm{B}^{1}\right)$ and the rest of the hypotheses of Theorem 1.1 are satisfied, then: (1) there exists a unique solution to equation (1.1); (2) this solution can be obtained by successive approximations.

Instead of establishing these results directly, it is more illuminating to present the basic reasoning upon which they depend in abstract form first and then to interpret it appropriately for equation (1.1).

LEMMA 1.1. If $U$ is a completely continuous linear operator over $L_{2}$ with values in $L_{2}$, and if $G$ is any continuous operator over $L_{2}$ with values in $L_{2}$ such that for some positive constant $A,\|G(\psi)\|<A+\gamma\|\psi\|$ for any $\psi$ in $L_{2}$, and if $\|U\|_{\gamma}=a<1$, then there exists at least one solution to $\psi=U G(\psi)$.

Consider the one-parameter family of equations:

$$
\psi=\tau U G(\psi)
$$$$
0 \leqq \tau \leqq 1 .
$$ 
For $\tau=0$, this reduces to the trivial equation $\psi=0$ which of course has a unique solution. For $\tau=1$, it becomes the equation of the lemma.

Furthermore, there exists an a priori bound, independent of $\tau$, to all solutions of equation (1.4) since

$$
\|\psi\| \leqq \tau\|U\|\|G(\psi)\| \leqq \tau\|U\|\{A+\gamma\|\psi\|\} \leqq\|U\| A+\|U\| \gamma\|\psi\| .
$$

Hence

$$
\|\psi\| \leqq \frac{\|U\| A}{1-\gamma\|U\|}
$$

According to Schauder-Leray [5], a solution to equation (1.4) will therefore exist for each value of $\tau$, hence for $\tau=1$.

In order to obtain a constructive process it is convenient to prove the following:

LEMMA 1.2. Let $U$ be a continuous linear operator defined over $L_{2}$ with values in $L_{2}$ and let $\left\{U_{n}\right\}$ be a sequence of continuous linear operators with the property that

$$
\Delta_{n}=\left\|U_{n}-U\right\| \rightarrow 0 \quad \text { as } n \rightarrow \infty .
$$

If $G$ is any continuous operator defined over $L_{2}$ with values in $L_{2}$ such that $\|G(x)-G(y)\| \leqq \gamma\|x-y\|$ where $\|U\| \gamma=a<1$, then there exists a unique solution to the equation $\psi=U G(\psi)$ which can be obtained by a successive approximation process based on $\psi_{0}$, an arbitrary element in $L_{2}$, and the equations $\psi_{n}$ $=U_{n} G\left(\psi_{n-1}\right)$.

Observe first that there exists an $n_{0}$ such that for $n>n_{0}$

$$
\left\|U_{n}\right\| \gamma \leqq b<1
$$

since

$$
\gamma\left\|U_{n}\right\| \leqq \gamma\left\|U-U_{n}\right\|+\gamma\|U\| \leqq \gamma \Delta_{n}+a
$$

and $\gamma \Delta_{n}$ can be made arbitrarily small.by taking $n$ sufficiently large.

Second, note that it follows that there exists a positive constant $A$ such that $\|G(\psi)\| \leqq A+\gamma\|\psi\|$ for $\|G(\psi)\| \leqq\|G(\psi)-G(0)\|+\|G(0)\| \leqq \gamma\|\psi\|+\|G(0)\|$. then

Third, the sequence $\left\{\psi_{n}\right\}$ is bounded (uniformly) in norm, for if $n>n_{0}$,

$$
\left\|\psi_{n}\right\| \leqq\left\|U_{n}\right\|\left\{A+\gamma\left\|\psi_{n-1}\right\|\right\} \leqq C+b\left\|\psi_{n-1}\right\|
$$

with $C=A b / \gamma$. Consequently, by induction

$$
\left\|\psi_{n}\right\| \leqq C+b C+\cdots+b^{n-n_{0}}\left\|\psi_{n_{0}}\right\| \leqq \frac{C}{1-b}+\left\|\psi_{n_{0}}\right\|=B
$$

Observe, finally, that the sequence $\left\{\psi_{n}\right\}$ converges to a point $\psi \in L_{2}$ which 
does satisfy equation (1.4) for $\tau=1$, since

$$
\begin{aligned}
\psi_{p}-\psi_{n} & =U_{p} G\left(\psi_{p-1}\right)-U_{n} G\left(\psi_{n-1}\right) \\
& =\left(U_{p}-U\right) G\left(\psi_{p-1}\right)+\left(U-U_{n}\right) G\left(\psi_{n-1}\right)+U\left[G\left(\psi_{p-1}\right)-G\left(\psi_{n-1}\right)\right]
\end{aligned}
$$

from which

$$
\begin{aligned}
\left\|\psi_{p}-\psi_{n}\right\| & \leqq \Delta_{p}\left\{A+\gamma\left\|\psi_{p-1}\right\|\right\}+\Delta_{n}\left\{A+\gamma\left\|\psi_{n-1}\right\|\right\}+a\left\|\psi_{p-1}-\psi_{n-1}\right\| \\
& \leqq(A+\gamma B)\left(\Delta_{p}+\Delta_{n}\right)+a\left\|\psi_{p-1}-\psi_{n-1}\right\| .
\end{aligned}
$$

Now choose $n_{1}$ so that $(A+\gamma B) \Delta_{n}<\epsilon / 2$ for $n>n_{1}$, and $n_{2}>n_{1}$ so that $n_{2}=n_{1}+k$ where $2 a^{k} B<\epsilon$; then let $n, p>n_{2}$, so that

$$
\begin{aligned}
\left\|\psi_{p}-\psi_{n}\right\| & \leqq \epsilon+a\left\|\psi_{p-1}-\psi_{n-1}\right\| \\
& \leqq \epsilon+a \epsilon+a^{2} \epsilon+\cdots+a^{k-1} \epsilon+a^{k}\left\|\psi_{p-k}-\psi_{n-k}\right\| \\
& \leqq \frac{\epsilon}{1-a}+2 a^{k} B \leqq \epsilon\left(\frac{1}{1-a}+1\right)
\end{aligned}
$$

from which it follows that there exists a $\psi$ such that $\left\|\psi-\psi_{n}\right\| \rightarrow 0$.

However, since

$$
U G(\psi)-\psi=U\left[G(\psi)-G\left(\psi_{n}\right)\right]+U G\left(\psi_{n}\right)-\psi_{n+1}+\psi_{n+1}-\psi,
$$

it follows that

$$
\begin{aligned}
\|U G(\psi)-\psi\| & \leqq a\left\|\psi-\psi_{n}\right\|+\left\|U G\left(\psi_{n}\right)-U_{n} G\left(\psi_{n}\right)\right\|+\left\|\psi_{n+1}-\psi\right\| \\
& \leqq a\left\|\psi-\psi_{n}\right\|+\Delta_{n}\{A+\gamma B\}+\left\|\psi_{n+1}-\psi\right\|
\end{aligned}
$$

from which $\psi=U G(\psi)$, since the right-hand side can again be made arbitrarily small.

In order to verify that the equation $\psi=U G(\psi)$ has a unique solution under these hypotheses, assume that two solutions $\psi_{1}, \psi_{2}$ of it existed. Then it would follow that

$$
\left\|\psi_{2}-\psi_{1}\right\|=\left\|U G\left(\psi_{2}\right)-U G\left(\psi_{1}\right)\right\| \leqq\|U\| \gamma\left\|\psi_{2}-\psi_{1}\right\|<\left\|\psi_{2}-\psi_{1}\right\|
$$

and consequently that $\left\|\psi_{2}-\psi_{1}\right\|=0$.

In order to apply these lemmas to establish Theorems 1.1 and 1.2, let

$$
\bar{\mu}=\frac{\mu_{N+1}+\mu_{N}}{2}, \quad \gamma=\frac{\mu_{N+1}-\mu_{N}}{2}
$$

and define the continuous function $g(x, y)$ by the relation:

$$
g(x, y)=f(x, y)-\bar{\mu} y \text {. }
$$

By hypothesis $f(x, y)$ satisfies $\left(\mathrm{B}^{1}\right)$. In terms of $g(x, y)$, this is:

$$
-\gamma y-A \leqq g(x, y) \leqq \gamma y+A \quad \text { for } y>y_{0},
$$




$$
\gamma y-A \leqq g(x, y) \leqq-\gamma y+A
$$

hence

$$
-\gamma|y|-A \leqq g(x, y) \leqq \gamma|y|+A \quad \text { for } y>\left|y_{0}\right| .
$$

Thus a constant $C$ can be found such that

$$
|g(x, y)| \leqq \gamma|y|+C
$$

for all $y$.

Now if $\psi(x)$ is any element in $L_{2}$, it follows that $g(x, \psi(x))$ defines a continuous operator $G(\psi)$ over $L_{2}$ with values in $L_{2}$. Further from the above inequalities on $g(x, y)$ it follows that

$$
\|G(\psi)\| \leqq\|\gamma|\psi(x)|+C\| \text {. }
$$

Thus a constant $D$ can be found such that

$$
\|G(\psi)\| \leqq \gamma\|\psi\|+D
$$

Let $K=\int_{a}^{b} K(x, y)$ [ ] $d y$ be the completely continuous Hermitian operator in $L_{2}$ of Theorem 1.1 ; let $\left\{\phi_{\rho}\right\}$ denote the complete ortho-normal set of characteristic functions of $K$ and let $\left\{\lambda_{\rho}\right\}$ denote the corresponding set of characteristic values of $K$, it being understood that in the case of multiple characteristic functions corresponding to a single characteristic value, the characteristic value will be considered as occurring with a corresponding multiplicity. Let the identity operator be denoted by $I$, the operator $K /(I-\bar{\mu} K)$ by $U$, the operation of projection into the linear subspace spanned by $\left\{\phi_{\rho}\right\}, \rho=N, N-1, \cdots(-\infty)$ by $P_{N}$, and the operators $P_{N} U$ by $U_{N}$. With these definitions the following lemma is obvious.

Lемма 1.3. (1) If $f(x, y)$ satisfies $\left(\mathrm{B}^{1}\right)$, then $\mathrm{G}$ satisfies

$$
\|G(\psi)\|<A+\gamma\|\psi\|
$$

for some constant $A$.

(2) If $(x, y)$ satisfies $\left(C^{1}\right)$, then $G$ satisfies

$$
\begin{aligned}
\left\|G\left(\psi_{2}\right)-G\left(\psi_{1}\right)\right\| & \leqq \gamma\left\|\psi_{2}-\psi_{1}\right\| . \\
\left\|U_{N}-U\right\| & \rightarrow 0 \quad \text { as } N \rightarrow \infty .
\end{aligned}
$$

In order to verify the norm condition of Lemmas 1.1 and 1.2 on $U$, it is first necessary to find the characteristic values of $U$.

LEMмA 1.4. The complete ortho-normal set of characteristic functions of the operator $K$ are also a complete ortho-normal set of characteristic functions for the linear operator $U$ and $\left\{\lambda_{\rho}-\bar{\mu}\right\}$ are the set of corresponding characteristic values.

Let $\psi_{n}$ be any characteristic function of $U$ with characteristic value $\sigma_{n}$ so 
that $\psi_{n}=\sigma_{n} U\left(\psi_{n}\right)$. By the definition of $U$ this is equivalent to: $\psi_{n}=\left(\sigma_{n}+\bar{\mu}\right) K \psi_{n}$. Therefore $\psi_{n}$ must be some characteristic function of $K$, say $\dot{\phi}_{n}$ with characteristic value $\lambda_{n}$. That is, $\lambda_{n}=\sigma_{n}+\bar{\mu}$ from which one has $\sigma_{n}=\lambda_{n}-\bar{\mu}$.

The fact that the above choice of $U$ and $\gamma$ implies that $\|\boldsymbol{U}\| \boldsymbol{\gamma}=a<1$ now follows immediately from the well known fact that

$$
\|U\|=\sup \left|\frac{1}{\sigma_{i}}\right|=\sup \left|\frac{1}{\lambda_{i}-\bar{\mu}}\right|<\frac{1}{\gamma} .
$$

Finally it is necessary to observe that the complete continuity of the operator $U$ follows from the complete continuity of $K$ and the fact that $K$ is the linear operator associated with the Hermitian kernel $K(x, y)$. That is, it follows from the theory of linear integral equations that since $\bar{\mu}$ is different from all of the characteristic values of $K$, the operator $(I-\bar{\mu} K)$ has a continuous inverse $(I+\bar{\mu} R)$ where $R$ is the linear operator associated with the resolvent kernel of $K(x, y)$. Thus $U=K /(I-\bar{\mu} K)=K(I+\bar{\mu} R)$ and since $K$ is by hypotheses completely continuous, it follows that $U$ is too.

Theorem 1.1 now follows immediately from these definitions and Lemma 1.1. Similarly, Theorem 1.2 follows from these definitions and Lemma 1.2.

\section{PART II}

Even a casual analysis of the preceding development reveals the fundamental role played by the a priori estimate given by $(1.5)$ whenever $\left(B^{1}\right)$ is satisfied. Unfortunately, if $\left(\mathrm{B}^{1}\right)$ is replaced by the corresponding generalization of (A), this estimate ceases to hold and the situation is neither so clear nor so simple.

In discussing (A) Hammerstein restricted himself to positive-definite kernels, made use of Mercer's representation $K(x, y)=\sum \phi_{i}(x) \phi_{i}(y) / \lambda_{i}$, and used the fact that the unknown function could be expressed as $\psi(x)$ $=\sum \alpha_{i} \phi_{i}(x)$ where

$$
\lambda_{i} \alpha_{i}=\int_{a}^{b} f\left[y, \sum \alpha_{j} \phi_{j}(y)\right] \phi_{i}(y) d y, \quad i=1,2, \cdots
$$

However, these last equations are nothing but the Euler-Lagrange equations for the functional

$$
J(\psi)=\sum \lambda_{i} \alpha_{i}^{2}-2 G\left(\sum \alpha_{i} \phi_{i}\right)
$$

where

$$
G(\phi)=\int_{a}^{b} \int_{0}^{\phi(x)} f(x, v) d v d x
$$

and it is easily seen that (A) is just sufficient to guarantee the existence of an infimum to $J(\psi)$ and to provide an a priori bound to $\|\psi\|=\left\{\sum_{i=1}^{\infty} \alpha_{i}^{2}\right\}^{1 / 2}$. 
The natural generalization of $(A)$ is

$$
\mu_{N} v^{2}-C_{N} \leqq 2 \int_{0}^{v} f(x, y) d y \leqq \mu_{N+1} v^{2}+C_{N+1}
$$

This requires that

$$
\sum_{i=1}^{\infty}\left(\lambda_{i}-\mu_{N+1}\right) \alpha_{i}^{2}-C_{N+1} \leqq J(\psi) \leqq \sum_{i=1}^{\infty}\left(\lambda_{i}-\mu_{N}\right) \alpha_{i}^{2}+C_{N}
$$

Condition $\left(\mathrm{A}^{1}\right)$ or (2.2) is not sufficient, however, to guarantee the existence of an a priori estimate as the following example shows.

Let $i$ be restricted to 1,2 , then (2.2) states that the surface $z=J(\psi)$ must always lie below the surface $S_{1}$

$$
z-C_{1}=-\left(\mu_{1}-\lambda_{1}\right) \alpha_{1}^{2}+\left(\lambda_{2}-\mu_{1}\right) \alpha_{2}^{2}
$$

and above the surface $S_{2}$

$$
z+C_{2}=-\left(\mu_{2}-\lambda_{1}\right) \alpha_{1}^{2}+\left(\lambda_{2}-\mu_{2}\right) \alpha_{2}^{2} .
$$

It is clear that saddle surfaces of the general form

$$
z=-a \alpha_{1}^{2}+b \alpha_{2}^{2}
$$

will satisfy this requirement for appropriately chosen values of $a, b$. However, an examination of the contour map of the $\alpha_{1}, \alpha_{2}$ plane of surfaces of this type reveals that it is possible to displace continuously the critical point, defined by $(2.1)$ for $i=1,2$, to plus infinity along the line $(a x=b y)$ without disturbing the contour lines of greater than $P$ or less than $P$ for any preassigned constant $P$. The deformed surface will therefore still satisfy $(2.2)$ for $i=1,2$ and will in fact possess no critical point not at infinity. This is of course equivalent to stating that equations (2.1) do not possess a bounded solution, hence surely not an a priori bound. This simple example makes it clear that under $\left(\mathrm{A}^{1}\right)$ there is no topological necessity for an a priori bound, though it must be admitted that the special analytical form of $J(\psi)$ may still permit such a bound to exist.

The ideas and methods of the calculus of variations in the large make it reasonable to seek existence proofs without recourse to a priori estimation and thus open an entirely new approach to nonlinear problems which can be associated with a variational problem. A first attempt at such a method will be outlined in the sequel and made to yield an existence theorem which permits the estimation of the norm of the solution only after its existence has been concluded (a posteriori estimation). In its present state the method is not completely satisfactory since it employs only a restricted class of cycles, namely linear, finite-dimensional manifolds. This severe restriction on the class of admissible cycles limits the class of permissible deformations and 
consequently more stringent hypotheses are required here then it is believed will be necessary when the restriction on the admissible class of cycles is removed.

Before outlining the method it will be convenient to transform the problem in a manner suggested by the work of Golomb [4]. Let $L_{2}$ be the Hilbert space consisting of all real quadratically integrable functions defined over the interval $a \leqq x \leqq b$ with an inner product defined in the usual way by means of

$$
\left(\phi_{1}, \phi_{2}\right)=\int_{a}^{b} \phi_{1}(x) \phi_{2}(x) d x
$$

for $\phi_{1}$ and $\phi_{2}$ in $L_{2}$. Let $K(x, y)$ be a continuous, positive-definite, and symmetrical kernel, $\left\{\phi_{\rho}(x)\right\}, \rho=1,2, \cdots$, the complete orthonormal system of its characteristic functions, and $\left\{\lambda_{\rho}\right\}, \rho=1,2, \cdots$, the corresponding set of characteristic values where the characteristic values are taken so that $0<\lambda_{1}<\lambda_{2}<\cdots$. The kernel $K(x, y)$ defines a bounded, linear, positivedefinite, completely continuous, and symmetric operator in the usual way by means of

$$
K \phi=\int_{a}^{b} K(x, y) \phi(y) d y=\sum_{\rho=1}^{\infty} \frac{\left(\phi, \phi_{\rho}\right)}{\lambda_{\rho}} \phi_{\rho} ;
$$

Similarly, the relation

$$
H \phi=\sum_{\rho=1}^{\infty} \frac{\left(\phi, \phi_{\rho}\right)}{\left(\lambda_{\rho} ; \rho\right)^{1 / 2}} \phi_{\rho}
$$

defines a bounded, linear, positive-definite, completely continuous, and symmetric operator with the properties that

$$
K \phi=H(H \phi)=H^{2} \phi
$$

and such that for some positive constant $C$

$$
|H \phi(x)| \leqq C\|\phi\|
$$

for each $\phi \in L_{2}$ and $a \leqq x \leqq b\left({ }^{3}\right)$.

Defining the operator $F(\psi)=f(y, \psi(y))$, for each $\psi$ in $L_{2}$, equation (1.1) can be replaced by the abstract functional equation

$$
\psi=K F(\psi) \text {. }
$$

This in turn can be replaced by the functional equation

$$
\phi=H F(H \phi)
$$

(3) For a more general treatment of the existence, uniqueness and complete continuity of the "square-root" operator $\boldsymbol{H}$ than that given by Golomb [4], see, for example, Nagy [9], where the discussion is based on the spectral theorem. 
as the next lemma will demonstrate. Equation (2.4) is more convenient for the sequel in that it leads to a more readily discussed variational problem than that for $J(\psi)$. The fact that a solution of equation (2.4) yields a solution to (2.3) was first noticed by Golomb [4].

Lemma 2.1. A necessary and sufficient condition that equation (2.3) possess a solution in $L_{2}$ where $K$ is a positive self-adjoint linear operator over $L_{2}$ with values in $L_{2}$ is that the functional equation (2.4) have a solution where $H$ is the unique, self-adjoint operator such that $K=H^{2}$.

Necessity. Let $\psi_{0}$ be a solution of equation (2.3), then

$$
\phi_{0}=H F\left(\psi_{0}\right)
$$

is a solution of (2.4) for

$$
\psi_{0}=K F\left(\psi_{0}\right)=H^{2} F\left(\psi_{0}\right)=H\left(H F\left(\psi_{0}\right)\right)=H \phi_{\theta} .
$$

The substitution of this last equality into equation (2.5) yields equation (2.4).

Sufficiency. Let $\phi_{0}$ be a solution of equation (2.4), then

$$
\psi_{0}=H \phi_{0}
$$

is a solution of equation (2.3) since equation (2.4) implies that

$$
H \phi_{0}=H\left(H F\left(H \phi_{0}\right)\right)=H^{2} F\left(H \phi_{0}\right)=K F\left(\psi_{0}\right) \text {. }
$$

The substitution of this last equality into equation (2.6) yields equation (2.3).

Recalling Golomb's [4] definition of the gradient of a functional; namely, an operator $\boldsymbol{\Gamma}$ defined in $L_{2}$ with values in $L_{2}$ will be called the gradient of a functional $G(\phi)$ if for any two different elements $\phi_{1}, \phi_{2}$ in $L_{2}$,

$$
G\left(\phi_{2}\right)-G\left(\phi_{1}\right)=\left(\Gamma \phi_{1}, \phi_{2}-\phi_{1}\right)+R\left(\phi_{1}, \phi_{2}\right)\left\|\phi_{2}-\phi_{1}\right\|
$$

holds where $R\left(\phi_{1}, \phi_{2}\right)$ is a continuous function for which

$$
\lim _{\phi_{2} \rightarrow \phi_{1}} R\left(\phi_{1}, \phi_{2}\right)=0
$$

and defining the function $G(x, v)=\int_{0}^{v} f(x, y) d y$ the operators $F(\phi)=f(y, \phi(y))$ and

$$
G(\phi)=\int_{a}^{b} G(x, \phi(x)) d x
$$

for all $\phi$ in $L_{2}$, we easily verify that the operator

$$
\boldsymbol{\Gamma} \phi=2[\phi-H F(H \phi)]
$$

is the gradient of the functional

$$
I(\phi)=(\phi, \phi)-2 G(H \phi) .
$$


Consequently, equation (2.4) will possess a solution $\phi_{0}$ if and only if $\boldsymbol{\Gamma} \phi_{0}$ $=\operatorname{grad} I\left(\phi_{0}\right)=0$.

Let $M_{N}^{0}$ denote the $N$-dimensional linear manifold in Hilbert space spanning the origin and $\phi_{1}, \cdots, \phi_{N}$, the first $N$ characteristic functions of the operator $K$. Let $\Omega$ denote the class of linear manifolds $\left\{M_{N}\right\}$ in Hilbert space which are parallel to $M_{N}^{0}$. Thus if one lets $\xi$ be the function in $M_{N}$ which is orthogonal to all $\phi$ in $M_{N}^{0}$ one has $M_{N}(\xi)=M_{N}^{0}+\xi$. With these definitions the main theorem of this section is the following:

THEOREM 2.1. (1) If $K$ is a (completely continuous) linear operator over $L_{2}$, with values in $L_{2}$, which is defined by the positive-definite, symmetric, and continuous kernel $K(x, y)$ by the relation

$$
K=\int_{a}^{b} K(x, y)[] d y
$$

and if $\left\{\lambda_{\rho}\right\}, \rho=1,2, \cdots$, denote the denumerable set of characteristic values of $K$ in the sense that $0<\lambda_{1}<\lambda_{2}<\cdots$; (2) If the functional

$$
G(\phi)
$$

satisfies the inequality

$$
\mu_{N}(\phi, \phi)-C_{N} \leqq 2 G(\phi) \leqq \mu_{N+1}(\phi, \phi)+C_{N+1}
$$

for all $\phi$ in $L_{2}$, where $C_{N}, C_{N+1}, \mu_{N}$, and $\mu_{N+1}$ are constants such that $C_{N} \geqq 0$, $C_{N+1} \geqq 0$, and $\lambda_{N}<\mu_{N}<\mu_{N+1}<\lambda_{N+1}$; (3) If the functional $I(\phi)$ defined by equation (2.10) possesses at most one maximum on each linear manifold $M_{N}$ in $\Omega$; then equation (1.1) possesses at least one solution.

Before discussing the proof, it should be remarked that the functional $I(\phi)$ will certainly satisfy condition (3) above if the functional $G(\boldsymbol{H} \phi)$ is a concave functional of $\phi$ over each $M_{N}$.

The proof will follow the familiar pattern of proofs given by methods in the large. Defining

$$
d\left(M_{N}\right)=\max _{\phi \in M_{N}} I(\phi)
$$

and

$$
d=\inf _{M_{N} \in \Omega} d\left(M_{N}\right)
$$

one first shows that $d$ is finite. Once this has been done, the existence of a limit manifold $M_{N}^{*}$ is demonstrated and it is shown that $d\left(M_{N}^{*}\right)=d$. By hypothesis, it follows that there exists a $\phi_{0} \in M_{N}^{*}$ such that $I\left(\phi_{0}\right)=d$. Finally, it is shown that unless $\phi_{0}$ satisfies equation (2.4), the limit manifold $M_{N}^{*}$ could be continuously displaced into a new linear manifold $M_{N}^{* *}$ on which $d\left(M_{N}^{* *}\right)<d$, 
contrary to the definition of $d$. Let $\phi$ be any point in any linear manifold $M_{N}$ belonging to $\Omega$. Then $\phi$ may be represented by

$$
\phi=\theta+\xi ; \theta \in M_{N}^{0} ; \theta=\sum_{i=1}^{N} \alpha_{i} \phi_{i} ; \quad(\theta, \xi)=0
$$

where, as is well known from linear theory,

$$
\begin{array}{rlrl}
\left(\phi_{i}, \phi_{j}\right) & =\delta_{i j} ;\left(\phi_{i}, \xi\right)=0, & & i=[1, \cdots, N \\
\left(H \phi_{i}, H \phi_{i}\right) & =\frac{1}{\lambda_{i}}\left(\phi_{i}, \phi_{i}\right), & i=1, \cdots, N \\
(H \xi, H \xi) & \leqq \frac{1}{\lambda_{N+1}}(\xi, \xi), \\
(H \theta, H \xi) & =\sum \alpha_{i}\left(H^{2} \phi_{i}, \xi\right) \\
& =\sum \frac{\alpha_{i}}{\lambda_{i}}\left(\phi_{i}, \xi\right)=0, \\
(H \theta, H \theta) & =\sum \frac{\alpha_{i}^{2}}{\left(H \phi_{i}, H \phi_{i}\right)} \\
& =\sum \frac{\alpha_{i}^{2}}{\lambda_{i}}
\end{array}
$$

These inequalities and $\left(\mathrm{A}^{2}\right)$ imply that

$$
\begin{aligned}
I(\phi) & \leqq(\theta, \theta)-\mu_{N}(H \theta, H \theta)+C_{N}+(\xi, \xi)-\mu_{N}(H \xi, H \xi), \\
I(\phi) & \leqq \sum_{i=1}^{N} \alpha_{i}^{2}\left(1-\frac{\mu_{N}}{\lambda_{i}}\right)+(\xi, \xi)+\mu_{N}(H \xi, H \xi)+C_{N} .
\end{aligned}
$$

However,

$$
\begin{gathered}
\left(1-\frac{\mu_{N}}{\lambda_{i}}\right) \leqq\left(1-\frac{\mu_{N}}{\lambda_{j}}\right)<0, \quad i \leqq j ; i=1, \cdots, N ; j=1, \cdots, N, \\
\left(1-\frac{\mu_{N+1}}{\lambda_{N+1}}\right)>0
\end{gathered}
$$

so that

$$
I(\phi) \leqq\left(1-\frac{\mu_{N}}{\lambda_{N}}\right)\|\theta\|^{2}+\left(1+\frac{\mu_{N}}{\lambda_{N+1}}\right)\|\xi\|^{2}+C_{N} .
$$

Similarly, the left-hand side of $\left(\mathrm{A}^{2}\right)$ implies that

$$
I(\xi) \geqq\left(1-\mu_{N+1} / \lambda_{N+1}\right)\|\xi\|^{2}-C_{N+1} .
$$

Several useful relations are immediate consequences of (2.11) and (2.12). Thus: 
For any $\phi$ contained in a given $M_{N}(\xi=$ constant $)$

$$
I(\phi) \rightarrow-\infty
$$

$$
\begin{aligned}
d\left(M_{N}\right) \geqq I(\xi) & \geqq\left(1-M_{N+1} / \lambda_{N+1}\right)\|\xi\|^{2}-C_{N+1} \geqq-C_{N+1}, \\
\|\xi\|^{2} & \leqq \frac{d\left(M_{N}\right)+C_{N+1}}{\left(1-\mu_{N+1} / \lambda_{N+1}\right)} \\
d\left(M_{N}^{0}\right) & \leqq\left(1-\mu_{N} / \lambda_{N}\right)\|\theta\|^{2}+C_{N} \leqq C_{N} .
\end{aligned}
$$

It follows from (2.14) and (2.16) that

$$
-C_{N+1} \leqq d \leqq C_{N}
$$

Let $\omega$ be defined as that subclass of $\Omega$ for which

$$
d\left(M_{N}\right) \leqq C_{N} .
$$

It is an immediate consequence of (2.15) that for any $M_{N}$ in $\omega$

$$
\|\xi\| \leqq\left\{\frac{C_{N}+C_{N+1}}{\left(1-\mu_{N+1} / \lambda_{N+1}\right)}\right\}^{1 / 2} .
$$

Consider, therefore, any sequence $\left\{M_{N}^{\alpha}\right\}$, such that $d M(\underset{N}{\alpha}) \rightarrow d$. It is clear from (2.16) that this sequence can be replaced without loss of generality by one involving only elements in $\omega$. Let $\xi_{\alpha}$ correspond to $M_{N}^{\alpha}$. Since any bounded set in Hilbert space is well known to be weakly compact $\left({ }^{4}\right)$, there will exist a subsequence $\left\{\xi_{\alpha}{ }^{\prime}\right\}$ of $\left\{\xi_{\alpha}\right\}$ which has a limit point $\xi_{0}$ in the weak topology $\left.{ }^{5}\right)$.

The limit point $\xi_{0}$ defines a linear manifold $M_{N}^{*}$ uniquely by

$$
M_{N}^{*}=M_{N}^{0}+\xi_{0} \text {. }
$$

Since $M_{N}^{*}$ is, by the above definition, included in $\Omega$, it follows by hypothesis that on it $I(\phi)$ attains its maximum at only one point, say $\phi_{0}$. Clearly

$$
I\left(\phi_{0}\right) \geqq d \text {. }
$$

Assume that $I\left(\phi_{0}\right)>d$; then it follows that there exists a subsequence of $N$-dimensional linear manifolds $\left\{M_{N}^{\beta}\right\} \in \omega$ such that $I\left(\phi_{0}\right)>d\left(M_{N}^{\beta}\right) \geqq d$. Let $\xi_{\beta}$ correspond to any $M_{N}^{\beta}$ and $\xi_{0}$ to $M_{N}^{*}$. Denote $\xi_{\beta}-\xi_{0}$ by $\Delta \xi_{\beta}$ so that $\phi_{0}+\Delta \xi_{\beta}$ will be on $M_{N}^{\beta}$, and

$$
\begin{aligned}
I\left(\phi_{0}+\Delta \xi_{\beta}\right)= & I\left(\phi_{0}\right)-2 G\left(H \phi_{0}+H \Delta \xi_{\beta}\right) \\
& +2 G\left(H \phi_{0}\right)+\left(\Delta \xi_{\beta}, \Delta \xi_{\beta}\right)+2\left(\phi_{0}, \Delta \xi_{\beta}\right) .
\end{aligned}
$$

However $\left\|\Delta \xi_{\beta}\right\|$ is bounded for all $\xi_{\alpha} \in M_{N} \in \omega$ and $H \phi_{0}$ is a continuous

(4) Imposing the condition $\left(\mathrm{A}^{2}\right)$ on the functional $I(\phi)$ is equivalent to imposing the condition $\left(\mathrm{A}^{1}\right)$ on the functional $J(\psi)$.

(5) See for example, F. H. Murray, Linear transformations in Hilbert space, Princeton University Press, chap. 5. 
function of $x$ on $(a, b)$ so that there exists constants $c, d$ such that on $(a, b)$

$$
c \leqq H \phi_{0}(x) \leqq d
$$

and

$$
c \leqq H \phi_{0}+H \Delta \xi_{\beta} \leqq d .
$$

From the continuity of $f[x, v(x)]$ it follows that there exists a constant $k$ such that

$$
|f[x, v(x)]| \leqq k
$$

for $a \leqq x \leqq b$ and $c \leqq v \leqq d$, and hence

$$
\begin{aligned}
\left|2 G\left(H \phi_{0}\right)-2 G\left(H \phi_{0}+H \Delta \xi_{\beta}\right)\right| & =2\left|\int_{a}^{b} \int_{H_{\phi_{0}}}^{H_{\phi_{0}}+H \Delta \xi_{\beta}} f[x, v(x)] d v d x\right| \\
& \leqq 2 k \int_{a}^{b}\left|H \Delta \xi_{\beta}\right| d x \leqq 2 k\left\|H \Delta \xi_{\beta}\right\|(b-a)^{1 / 2}
\end{aligned}
$$

Since $H$ is a completely continuous operator there exists a subsequence $H \Delta \xi_{\beta^{\prime}}$ which converges strongly. Moreover, since $\left(\phi_{0},\right)$ defines a linear functional in $L_{2}$, it follows from the weak convergences of $\Delta \xi_{\alpha^{\prime}} \rightarrow 0$ (hence a fortiori $\left.\Delta \xi_{\beta^{\prime}} \rightarrow 0\right)$ that $\left(\phi_{0}, \Delta \xi_{\beta^{\prime}}\right) \rightarrow 0$. Since the sequence $\xi_{\alpha^{\prime}}-\xi_{0}$ may be replaced by the sequence $\Delta \xi_{\beta^{\prime}}=\xi_{\beta^{\prime}}-\xi_{0}$ without loss of generality, it follows that

$$
\begin{aligned}
d\left(M_{N}^{\beta^{\prime}}\right) & =\max _{\phi \in M_{N} \beta^{\prime}} I(\phi) \geqq I\left(\phi_{0}+\Delta \xi_{\beta^{\prime}}\right) \\
& \geqq I\left(\phi_{0}\right)-2 k(b-a)^{1 / 2}\left\|H \Delta \xi_{\beta^{\prime}}\right\|+2\left(\phi_{0}, \Delta \xi_{\beta^{\prime}}\right) .
\end{aligned}
$$

Upon letting $\xi_{\beta^{\prime}} \rightarrow \xi_{0}$, this yields

$$
d \geqq I\left(\phi_{0}\right) \text {. }
$$

This and equation (2.19) imply that

$$
I\left(\phi_{0}\right)=d
$$

Now if $\phi_{0}$ does not satisfy equation (2.4), then $\operatorname{grad} I\left(\phi_{0}\right) \neq 0$ so that $\eta=\operatorname{grad} I\left(\phi_{0}\right) /\left\|\operatorname{grad} I\left(\phi_{0}\right)\right\|$ defines a unit vector in Hilbert space. Now it is easy to see that grad $I\left(\phi_{0}\right)$ and hence $\eta$ must be orthogonal to $M_{N}^{*}$. For otherwise $\operatorname{grad} I\left(\phi_{0}\right)$ would have a projection on $M_{N}^{*}$. Let $u$ be a unit element in the direction of this projection. Then for all $t, 0 \leqq t \leqq \infty$, it follows from the hypothesis that $\phi_{0}$ is the only maximum of $I(\phi)$ on $M_{N}^{*}$ and the definition of the gradient that

$$
0 \geqq I\left(\phi_{0}+t u\right)-I\left(\phi_{0}\right)=t\left(\operatorname{grad} I\left(\phi_{0}\right), u\right)+R\left(\phi_{0}, \phi_{0}+t u\right)\|t u\|
$$

and that 


$$
0 \geqq I\left(\phi_{0}-t u\right)-I\left(\phi_{0}\right)=-t\left(\operatorname{grad} I\left(\phi_{0}\right), u\right)+R\left(\phi_{0}, \phi_{0}-t u\right)\|-t u\| .
$$

Dividing by $t$ and taking the limit as $t \rightarrow 0$, these yield respectively

$$
\left(\operatorname{grad} I\left(\phi_{0}\right), u\right) \leqq 0
$$

and

$$
\left(\operatorname{grad} I\left(\phi_{0}\right), u\right) \geqq 0
$$

from which it follows that ( $\left.\operatorname{grad} I\left(\phi_{0}\right), u\right)=0$.

Moreover $\operatorname{grad} I(\phi)$ is a continuous function of $\phi$ on $M_{N}^{*}$ so that there exists a positive number $e$ and a neighborhood $v_{0}$ of $\phi_{0}$ on $M_{N}^{*}$ such that for all $\phi \in v_{0}$

$$
(\operatorname{grad} I(\phi), \eta)>2 e>0 \text {. }
$$

Since $I(\phi)$ possesses only one maximum $\phi_{0}$ on $M_{N}^{*}$, there exists a positive number $\delta$ such that

$$
I(\phi)<I\left(\phi_{0}\right)-\delta
$$

for all $\phi$ on $M_{N}^{*}$ outside of $v_{e}$.

Now form the linear path in Hilbert space given by

$$
\sigma_{t}=\sigma_{0}-t \eta
$$

where $\sigma_{0}$ is an arbitrary element of $M_{N}^{*}$. Then by the definition of the gradient

$$
I\left(\sigma_{t}\right)-I\left(\sigma_{0}\right)=-t\left(\operatorname{grad} I\left(\sigma_{0}\right), \eta\right)+R\left(\sigma_{0}, \sigma_{t}\right)\|-t \eta\|
$$

where $\nu$ can be so chosen so that, for $0 \leqq t \leqq \nu$,

$$
\boldsymbol{R}\left(\sigma_{0}, \sigma_{t}\right)\|-t \eta\|<e .
$$

With this choice for $\nu$, it follows that whenever $\sigma_{0} \in v_{e}$

$$
I\left(\sigma_{t}\right)<I\left(\sigma_{0}\right)-e t \leqq I\left(\phi_{0}\right)-e t .
$$

Now define $s_{t}(0 \leqq t \leqq \nu)$ as that region of the functions

$$
\sigma_{t}=\theta+\xi_{0}-t \eta
$$

where $\theta \in M_{N}^{0}$ and

$$
\|\theta\|^{2} \leqq \frac{C_{N}-d+1+\left(1+\mu_{N} / \lambda_{N+1}\right)\left(\left\|\xi_{0}\right\|^{2}+\nu^{2}\|\eta\|^{2}\right)}{\mu_{N} / \lambda_{N}-1} .
$$

It follows from (2.11) that

$$
I\left(\sigma_{t}\right)<d-1
$$

whenever $\sigma_{0} \notin s_{0}$. Since $I(\phi)$ is a continuous function of the $N+1$ variables $t$, $\alpha_{i}(i=1, \cdots, N)$ over the bounded, hence compact, region $s_{t}, 0 \leqq t \leqq \nu$, it 
follows that for $\delta$ there exists a $\rho>0$ such that if $0 \leqq t \leqq \rho$, then

$$
\left|I\left(\sigma_{t}\right)-I\left(\sigma_{0}\right)\right|<\delta / 2
$$

whenever $\sigma_{0} \in s_{0}-v_{e}$. However this last inequality implies that if $\sigma_{0} \in s_{0}-v_{e}$, then

$$
I\left(\sigma_{t}\right)<I\left(\sigma_{0}\right)+\delta / 2 .
$$

Combining this with (2.21) one finds that if $\sigma_{0} \in s_{0}-v_{e}$, then

$$
I\left(\sigma_{t}\right)<I\left(\phi_{0}\right)-\delta / 2 \text {. }
$$

Consider the parallel displacement given by (2.22) of $M_{N}^{*}$ into $M_{N}^{* *}=\mu_{N}\left(t_{0}\right)$ where $t_{0}=\min (\rho, \nu)$. As a result of it, one has

$$
d\left(M_{N}^{* *}\right)<d=I\left(\phi_{0}\right)
$$

for:

(1) If $\sigma_{0} \in v_{e}$ then, since $t_{0} \leqq \nu$, equation (2.23) implies that

$$
I\left(\sigma_{t_{0}}\right) \leqq I\left(\phi_{0}\right)-e t
$$

(2) If $\sigma_{0} \in s_{0}-v_{e}$ then, since $t_{0} \leqq \rho$, equation (2.25) implies that $I\left(\sigma_{t_{0}}\right)$ $<I\left(\phi_{0}\right)-\delta / 2$.

(3) If $\sigma_{0} \notin s_{0}-v_{e}$ and $\sigma_{0} \notin v_{e}$, then equation (2.24) implies that

$$
I\left(\sigma_{t_{0}}\right)<d-1
$$

Since (2.26) cannot hold according to the definition of $d$, as $M_{N}^{* *}$ is clearly an admissible element of $\Omega$, it follows that grad $I\left(\phi_{0}\right)=0$ which implies that $\phi_{0}$ is a solution of equation (2.4). Lemma 2.1 now implies that, under the conditions of Theorem 2.1, equation (1.1) has at least one solution.

The norm of this solution can now be estimated as follows (a posteriori estimation):

Since $\phi_{0}=\theta_{0}+\xi_{0}$, it follows from (2.11), (2.17), and (2.201) that

$$
-C_{N+1} \leqq d=I\left(\phi_{0}\right)<\left(1-\mu_{N} / \lambda_{N}\right)\left\|\theta_{0}\right\|^{2}+\left\|\xi_{0}\right\|^{2}\left(1+\mu_{N} / \lambda_{N+1}\right)+C_{N}
$$

and hence that

$$
\left\|\theta_{0}\right\|^{2}<\frac{C_{N}+C_{N+1}+\left\|\xi_{0}\right\|^{2}\left(1+\mu_{N} / \lambda_{N+1}\right)}{\left(\mu_{N} / \lambda_{N}-1\right)} .
$$

However, by (2.15)

$$
\left\|\xi_{0}\right\|^{2} \leqq \frac{\left(C_{N}+C_{N+1}\right)}{\left(1-\mu_{N+1} / \lambda_{N+1}\right)}
$$

so that 


$$
\begin{aligned}
\left\|\phi_{0}\right\|^{2}=\left\|\theta_{0}\right\|^{2}+\left\|\xi_{0}\right\|^{2} & \leqq \frac{\left(C_{N+1}+C_{N}\right) \lambda_{N+1}}{\left(\lambda_{N+1}-\mu_{N+1}\right)}\left[1+\frac{\lambda_{N}\left(2 \lambda_{N+1}+\mu_{N}-\mu_{N+1}\right)}{\lambda_{N+1}\left(\mu_{N}-\lambda_{N}\right)}\right] \\
& \leqq \frac{\left(C_{N}+C_{N+1}\right) \lambda_{N+1}}{\left(\lambda_{N+1}-\mu_{N+1}\right)} \frac{\left(\mu_{N}+\lambda_{N}\right)}{\left(\mu_{N}-\lambda_{N}\right)} \\
& \leqq \frac{2\left(C_{N}+C_{N+1}\right)}{\left(1-\mu_{N+1} / \lambda_{N+1}\right)\left(1-\lambda_{N} / \mu_{N}\right)}
\end{aligned}
$$

which is the desired a posteriori estimate for equation (2.4). Similarly, by virtue of Lemma 2.1 and known bound of $H$ the a posteriori estimate for equation (1.1) can be readily found.

\section{PART III}

The above theorems will now be applied in their two-dimensional form to a boundary value problem in elliptical partial differential equations. (Cf. Hammerstein [1].)

THEOREM 3.1. (1) Let $B$ be a region of the $x, y$ plane in which the following linear problem has a solution( $\left.{ }^{(}\right)$.

$$
\begin{array}{cr}
L(v)=\frac{\partial}{\partial x}\left(p \frac{\partial v}{\partial x}\right)+\frac{\partial}{\partial y}\left(p \frac{\partial v}{\partial y}\right)=0 & \text { in } B, \\
v=\gamma & \text { on the boundary of } B ;
\end{array}
$$

(2) Let $L(v)$ possess a Green's function $K(x, y, s, t)$ that vanishes identically on the boundary of $B$ and is such that

$$
\iint_{B} K(x, y, s, t)[] d s d t
$$

is a completely continuous operator in the Hilbert space $L_{2}(B)$. Then in order that the nonlinear problem

$$
\begin{aligned}
L(u) & =f(x, y, u) \\
u & =\gamma
\end{aligned}
$$

in $B$, on the boundary of $B$

have a solution, it is sufficient that the function

$$
f(x, y, u+v)
$$

satisfy the hypotheses of Theorem 1.1, Theorem 1.2, or Theorem 2.1. In order for the solution to be unique it is sufficient for $f(x, y, u+v)$ to satisfy the hypothesis of Theorem 1.2.

As is well known, the boundary-value problem

${ }^{(6)}$ For a complete discussion of the existence of the solution to linear problems of this type reference can be made to Courant-Hilbert, Methoden der Mathematischen Physik, chap. 7, vol. 2. 


$$
L(\psi)=f[x, y, \psi(x, y)+v(x, y)]
$$

with

$$
\psi=0
$$

on the boundary of $B$

is equivalent to the integral equation

$$
\psi(x, y)=\iint_{B} K(x, y, s, t) f[s, t, \psi(s, t)+v(s, t)] d s d t .
$$

This equation is in the form of (1.1). Hence Theorems (1.1) or (1.2) or (2.1) may be applied to guarantee the existence (and perhaps uniqueness) of a solution. Now let

$$
u(x, y)=\psi(x, y)+v(x, y)
$$

where $v(x, y)$ is the solution of the linear problem stated in the hypotheses of the theorem. Then $u(x, y)$ is the solution of the nonlinear problem of the theorem, since

$$
\begin{array}{rlr}
L(u) & =L(\psi)+L(v)=L(\psi)=f(x, y, \psi+v) & \text { in } B, \\
u & =\psi+v=v=\gamma & \text { on the boundary of } B .
\end{array}
$$

The results of this paper may also be applied to the so-called generalized equation of Duffing which arises in the nonlinear theory of vibration. This equation may be written (cf. Hammerstein [1]) as

$$
\psi(x)+\lambda \int_{0}^{\pi} K(x, y) \sin \{-\psi(y)+g(y)\} d y=0
$$

where

$$
K(x, y)=\frac{2}{\pi} \sum_{n=1}^{\infty} \frac{\sin n x \sin n \pi}{n^{2}}=\left\{\begin{array}{lll}
x(-y / \pi+1) & \text { if } & x<y \\
y(-x / \pi+1) & \text { if } & x>v
\end{array}\right.
$$

and where $g(x)$ is assumed continuous. If we let

$$
f(x, u)=-\lambda \sin [u+g(x)]
$$

it follows that

$$
-|\lambda| \leqq f(x, u) \leqq|\lambda|
$$

so that the hypotheses of Theorem 1.1 are satisfied for all values of $\lambda$. Hence the generalized equation of Duffing possesses at least one solution for all values of $\lambda$. It is also easy to see that this equation fails to satisfy the sufficient condition for uniqueness $\left({ }^{7}\right)$.

( ${ }^{7}$ For an existence proof based upon the theory of E. Schmidt and the ideas of continuation and a discussion of the solutions of the generalized equation of Duffing see Iglisch [3]. 


\section{BIBLIOGRAPHY}

1. A. Hammerstein, Nichtlinear Integralgleichungen nebst Anwendungen, Acta Math. vol. 54 (1930) pp. 117-176.

2. R. Iglisch, Existence und Eindeutigkeit Sätze bei nichtlinear Integralgleichungen, Math. Ann. vol. 108 (1933) p. 161.

3. — Zur Theorie der Schwingungen, Monatshefte für Mathematik vol. 42 (1935) p. 7.

4. M. Golomb, Zur Theorie der nichtlinear Integralgelichungen, Integralgleichungsysteme und allgemeinen Functionalgleichungen, Math. Zeit. vol. 39 (1935) pp. 45-75.

5. Jean LeRay and Jules Schauder, Topologie et equations fonctionelles, Ann. Ecole Norm. vol. 51 (1934) pp. 45-68.

6. L. Lusternik and L. Schnirelmann, Methodes topologiques dans les problemes variationnels, Actualités Scientifiques et Industrielles, vol. 188, 1934.

7. F. Smithies, Completely continuous operators in Hilbert space, Bull. Amer. Math. Soc. vol. 44 (1938) p. 835.

8. T. H. Hildebrandt and L. M. Graves, Implicit functions and their differentials in general analysis, Trans. Amer. Math. Soc. vol. 29 (1927) pp. 127-153.

9. Belva v. Sz. Nagy, Spektraldarstellung linearer Transformationen des Hilbertschen Raumes, Ergebnisse der Mathematik, Ann Arbor, Edwards Brothers, 1947.

10. C. L. Dolph, Non-linear equations of the Hammerstein type, Proc. Nat. Acad. Sci. U.S.A. vol. 31 (1945) pp. 60-65.

\section{UNIVERSITY OF MichigAN,}

ANN ARBor, Mich.

Princeton University,

Princeton, N. J. 